\title{
LEIOMIOSARCOMA INTESTINAL
}

\section{LEIOMYOSARCOMA INTESTINAL}

\section{Cabello Fernández A, León Luque M, Maraver Zamora M, Bejarano García A}

Hospital Juan Ramón Jiménez. Huelva.

\section{Resumen}

El leiomiosarcoma digestivo es una neoplasia poco común, siendo el lugar de asentamiento más frecuente el estómago, seguido del intestino delgado (íleon). Sólo el $3 \%$ de los casos afectan al colon. En este último caso habría que hacer un diagnóstico diferencial fundamentalmente con el adenocarcinoma de colon. Su diagnóstico es histológico con necesidad de inmunohistoquímica, y su tratamiento curativo consiste en la cirugía, habiendo demostrado la quimio y radioterapiapocobeneficio.Supronósticoespobre,yaquela recidivatras la cirugía y la diseminación hematógena son frecuentes.

Palabras clave: Leiomiosarcoma intestinal, dolor abdominal, cirugía.
Abstract

Gastrointestinal leiomyosarcoma is a rare neoplasm, the stomach being the most frequent site of settlement, followed by the small intestine (ileum). Only $3 \%$ of cases affect the colon. In the latter case, a differential diagnosis would have to be made, fundamentally with colon adenocarcinoma. Its diagnosis is histological with the need for immunohistochemistry, and its curative treatment consists of surgery, with chemo and radiotherapy having shown little benefit. Its prognosis is poor, since recurrence after surgery and hematogenous dissemination are frequent.

Keywords: Intestinal leiomyosarcoma, abdominal pain, surgery.

\section{Introducción}

El leiomiosarcoma es una neoplasia rara que se origina en las células del músculo liso de las vísceras ${ }^{1}$. El leiomiosarcoma digestivo es más común en el estómago; en el intestino delgado afecta más frecuentemente el íleon. De todas las del tracto gastrointestinal sólo el 3\% está en colon. Supone menos del $1 \%$ de los tumores malignos colorrectales ${ }^{3}$. 
Su sintomatología es inespecífica: dolor abdominal y alteración del patrón evacuatorio y se puede palpar una masa abdominal a la exploración. Menos comunes son sus complicaciones: hemorragia, perforación y obstrucción.

\section{Caso Clínico}

Presentamos el caso de un varón de 70 años, fumador. Como único antecedente médico presenta psoriasis.

Acude a urgencias derivado por su médico de atención primaria por intensificación del cuadro de dolor abdominal de meses de evolución, en esta ocasión de predominio en fosa iliaca derecha e hipogastrio agudizado en los últimos días. Refiere además náuseas y vómitos así como hábito deposicional escaso y diarreico. El paciente refiere síntomas en el contexto de un síndrome constitucional asociado al cuadro de dolor abdominal crónico.

A la exploración se aprecia distensión abdominal y masa abdominal dolorosa a la palpación en fosa iliaca derecha.

Se realiza TC abdominal en urgencias (Figura 1) en el que se aprecia tumoración de $14 \mathrm{~cm}$ de diámetro en fosa ilíaca derecha que parece depender de intestino delgado, originando una obstrucción incompleta intestinal.

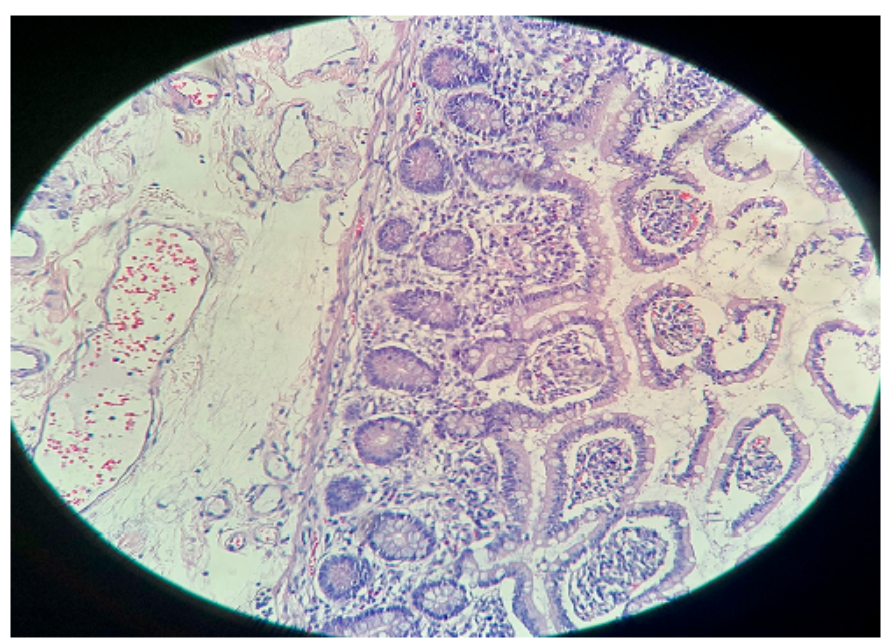

Figura 1

Diagnóstico de Leiomiosarcoma grado 3 de la FNCLCC. Estadio T2bG3NxM.

Es valorado por Cirugía de guardia que decide intervenir de urgencia.

El paciente es sometido a una hemicolectomía derecha ampliada con resección en bloque de yeyuno distal e íleon y resección de implantes mesentéricos con anastomosis latero-lateral.

El informe anatomopatológico describe una gran tumoración dependiente de intestino delgado, colón derecho y mesenterio. No se reconocen ganglios linfáticos en la muestra (Figura 2).

El patrón inmunohistoquimico confirma que se trata de un sarcoma de diferenciación muscular lisa (CKIT-;Dog1-;CD34-;

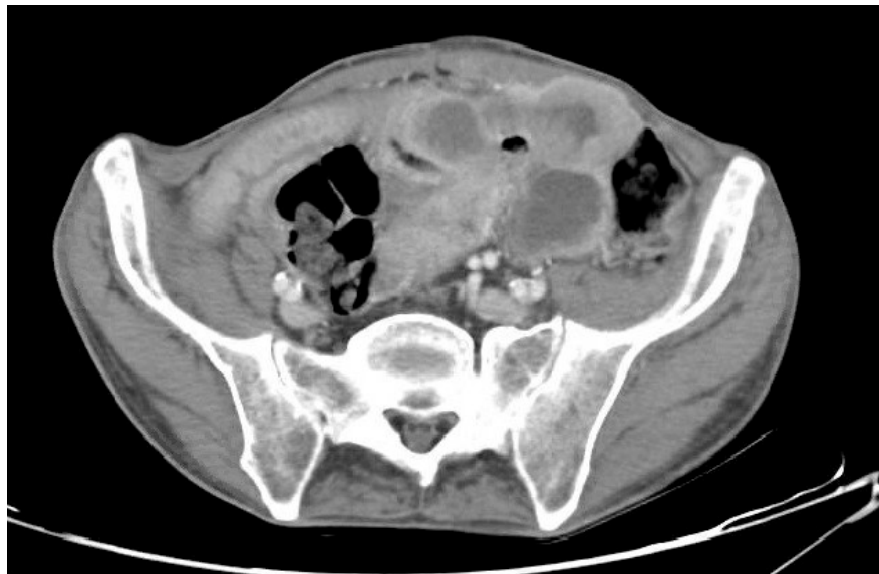

Figura 2

Tumoración de $14 \mathrm{~cm}$ en fosa ilíaca derecha.

Desmesina+; Actina+; Caldesmón+). La neoplasia infiltra la pared muscular de intestino delgado y grueso, respetando la mucosa. Diagnóstico de Leiomiosarcoma grado 3 de la FNCLCC. Estadio T2bG3NxM.

En comité de tumores se decide valoración por Oncología Médica que pauta tratamiento quimioterápico.

Pasados 4 meses de la intervención y tras iniciar quimioterapia acude a urgencias con dolor intenso en hemiabdomen inferior desde hace dos días asociado a deposiciones diarreicas de escasa cuantía.

Abdomen poco depresible con masa palpable en hemiabdomen inferior, doloroso y con signos de irritación peritoneal.

Se le realiza TC en el que se confirma obstrucción intestinal de alto grado con afectación retrógrada desde íleon, secundaria a diseminación neoplásica peritoneal y mesentérica. Además se

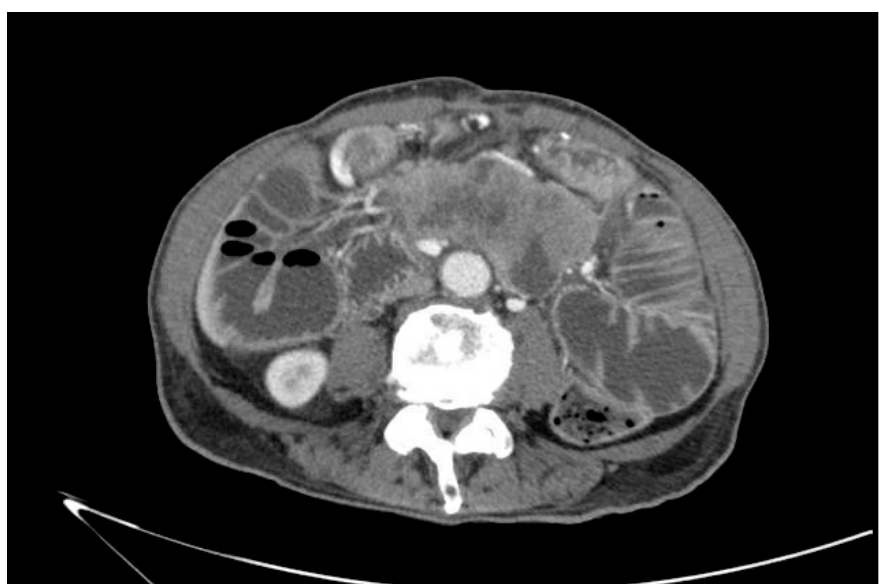

Figura 3

Obstrucción intestinal de alto grado con afectación retrógrada desde íleon, secundaria a diseminación neoplásica peritoneal y mesentérica. Además se observan lesiones hepáticas de nueva evolución. 
observan lesiones hepáticas de nueva evolución (Figura 3). Se desestima la intervención.

\section{Discusión}

El diagnóstico del leiomiosarcoma se basa en el estudio histológico e inmunohistoquímico de la pieza quirúrgica.

La cirugía es el tratamiento de elección. La recidiva tras cirugía radical se sitúa en torno al $40 \%$. La diseminación metastásica se produce fundamentalmente por vía hematógena, siendo el hígado el lugar de asiento más frecuente ${ }^{2}$.

La radioterapia y la quimioterapia no han demostrado eficacia en el tratamiento de este tipo de tumores.

Es importante incluir esta entidad en el diagnóstico diferencial de la neoplasia de colon así como la rápida actuación en diagnóstico y terapéutica quirúrgica dado que la cirugía es el tratamiento de elección y existen pobres alternativas de tratamiento médico ${ }^{4}$.

\section{Bibliografía}

1. Pilliado Páez H,Charua Guindic L, Avendaño Espinosa,Montes Villalobos J. Leiomiosarcoma colorrectal. Reporte de dos casos. An Med Asoc Med Hosp ABC 2000; 45(3): 140-4.

2. Flores Pastor B, Pellicer Franco E-Navarro Martínez MN, Carrasco González L-Aguayo Albasini JL. Metástasis hepáticas secundarias a leiomiosarcoma de recto. Presentación de un caso. Cir Esp 2000; 67: 394-

3. Mata JF, Escalante R, Linares K, Zamora M, Bassano L. Leiomyosarcoma of the gastrointestinal tract. GEN 1993; 47(1): $35-$ 44.

4. Fallahzadeh $\mathrm{H}$. Leiomyosarcoma of colon: report of two cases. Am Surg 1995; 61(4): 294-6. 